\title{
Thermally-induced optical reflection of sound (THORS) for photoacoustic sensing
}

Daniel S. Kazal, Ellen L. Holthoff, Brian M. Cullum

Daniel S. Kazal, Ellen L. Holthoff, Brian M. Cullum, "Thermally-induced optical reflection of sound (THORS) for photoacoustic sensing," Proc. SPIE 11020, Smart Biomedical and Physiological Sensor Technology XV, 1102009 (2 May 2019); doi: 10.1117/12.2517971

Event: SPIE Defense + Commercial Sensing, 2019, Baltimore, Maryland, United States 


\title{
Thermally-induced optical reflection of sound (THORS) for photoacoustic sensing
}

\author{
Daniel S. Kazal ${ }^{\mathrm{a}}$, Ellen L. Holthoff ${ }^{\mathrm{b}}$, Brian M. Cullum ${ }^{* a}$ \\ ${ }^{a}$ Dept. of Chemistry, University of Maryland, Baltimore County, 1000 Hilltop Cir., Baltimore, MD \\ USA 21250; U.S. Army Research Laboratory, 2800 Powder Mill Rd, Adelphi, MD USA 20783
}

\begin{abstract}
The ability to precisely control and manipulate acoustic waves can be highly limiting in applications and environments where placement of physical barriers for acoustic steering cannot be employed (e.g. tissues, air, etc.) In this work, we describe the ability to generate acoustic waveguides via thermally-induced optical reflection of sound (THORS) for the manipulation of acoustic waves in free space (i.e., air). Abrupt, density barriers are formed by photothermally depleting the sample in a laser beam's path via photothermal processes, resulting in sharp differences in compressibility and significant acoustic reflection (greater than 30\%). Optical waveguiding of sound can be achieved by generating THORS channels with a cylindrical (ring shaped) laser beam. By containing the acoustic waves inside a THORS cylindrical channel, a dramatically reduced acoustic decay profile of $1 / \mathrm{r}^{0.6}$ with distance is achieved.

Additionally, we describe the effects that optical modulation frequency of the THORS channel has on the efficiency of acoustic waveguiding. We also show how external acoustic waves, incident to a THORS channel are suppressed, increasing the signal-to-background ratio of the internally waveguided acoustic signals. Optical waveguiding of acoustic waves offers a new paradigm in the manipulation of sound over extended distances, providing potentially significant improvements to photoacoustic sensing, secure communications, and many other applications.
\end{abstract}

Keywords: Photoacoustic, Photothermal, THORS, Standoff sensing, Photoacoustic sensing

\section{INTRODUCTION}

Precise control and manipulation of acoustic waves are often highly limited to the applications and environments where placement of physical barriers for acoustic steering to be employed. This ability to manipulate acoustic waves is critically important to many scientific and engineering fields, including: subsurface tissue imaging, secure communications, acoustic design engineering, stealth technology, and photo-acoustic sensing. To achieve this acoustic manipulation, physical material interfaces are traditionally employed, where the magnitude and direction of the reflected, refracted and transmitted portions of the incident sound waves are controlled through differences in compressibility of the dissimilar acoustic transport media and the shape of the interface (relative to the incident acoustic wave). While the employment of physical material barriers provides an effective and predictable means for manipulation sound waves, they can require extensive time and effort to construct.

John Tyndall discovered an alternative method for manipulating sound waves in the 1860's, by showing that sound waves propagating through a flame, significantly decreased in amplitude on the other side, revealing that the differences in air density associated with the hot air through which the sound had passed dramatically affected the sounds transmission. ${ }^{1}$ Additionally, Tyndall's early work in sound propagation demonstrated that more efficient sound suppression occurred when propagating sound through a series of thinner slotted burners, as opposed to the single larger burner previous experiments were performed with., ${ }^{1,2}$ The mechanism by which the acoustic suppression occurred (i.e., refraction of the incident sound, reflection of the incident sound, etc.) was not characterized beyond a brief phenomenological description

Smart Biomedical and Physiological Sensor Technology XV, edited by Brian M. Cullum,

Douglas Kiehl, Eric S. McLamore, Proc. of SPIE Vol. 11020, 1102009 • (c) 2019

SPIE $\cdot$ CCC code: $0277-786 \mathrm{X} / 19 / \$ 18 \cdot$ doi: $10.1117 / 12.2517971$

Proc. of SPIE Vol. 11020 1102009-1 
of the effect. Alexander Graham Bell's subsequent discovery of the photoacoustic effect in the 1880's provided additional insights and a much clearer relationship between sound, heat, and light, in which the absorption of modulated light from the sun generated photo-thermal expansion and subsequent rarefaction of the absorbing material, thereby generating an audible sound. ${ }^{3,4}$ Largely forgotten until the 1970's, when the advent of intense laser sources, photoacoustic spectroscopy and its related family of photothermal spectroscopies (e.g., photothermal lensing) have since been employed for numerous applications, ranging from trace chemical monitoring, to medical diagnostics and imaging. ${ }^{5}$ With these photothermal techniques, localized density differences are generated in illuminated samples, and measured by either the generation of sound waves (i.e., photoacoustic spectroscopy) or the deviation of optical beams associated with the local difference in refractive index between the molecularly dense and rarefied regions (i.e., photothermal lensing/photothermal deflection). ${ }^{6}$ The potentially large optical absorptivity of the analyte molecules being detected and the efficient nature of non-radiative relaxation in molecules, results in these detection methodologies being extremely sensitive.

In this work, we characterize the physical characteristics of the recently discovered phenomenon, known as Thermallyinduced Optical Reflection of Sound (THORS) and demonstrate its potential for use as an acoustic waveguide for increasing the propagation distance of acoustic waves without the need for physical material interfaces, for improved photoacoustic sensing at extended distances. THORS is possible due to rapidly localized air density barriers generated by infrared excitation, acting as efficient acoustic reflectors along the beam path for any incident sound waves. By spatially and temporally shaping the beam into a cylindrical (donut-shaped) channel, THORS can be employed as an acoustic waveguide, internally reflecting acoustic waves down the channel's path to extend the overall propagation distance of sound waves in air, with dramatically reduced amplitude losses, when compared to non-confined acoustic waves. The extended sound wave propagation distance due to THORS channeling, provides the unique ability to dramatically increase the maximum detection distance or increase the spatial resolution at which photoacoustic sensing or imaging can be performed.

Previous studies of THORS have demonstrated that optically-induced photothermal barriers, generated in the same $\mathrm{CO}_{2}$ laser-based setup described in this work, can efficiently reflect a single, fixed-frequency soundwave propagating across the beam when monitored by a hearing aid microphone, smaller than the THORS barrier. ${ }^{7}$ Reflection of efficiencies large as $30 \%$ have been measured for incident sound waves ranging from $0 \mathrm{~dB}$ to $70 \mathrm{~dB}$ in amplitude, when propagating across a single THORS barrier. Additionally, this initial work has also demonstrated that when propagating soundwaves across multiple consecutive beams, the acoustic amplitude of the propagating soundwaves is suppressed completely.

The acoustic reflection of soundwaves incident to THORS barriers is understood to occur due to the abrupt difference in air density between the inside and outside edges of the laser beams path. The acoustic reflection efficiency can be mathematically described with classical acoustic physics as:

$$
\text { Reflection }=\frac{Z_{1}-Z_{2}}{Z_{1}+Z_{2}}
$$

where the difference in acoustic impedance, $Z_{1}$ and $Z_{2}$, of the air outside of the beam path and the air inside the beam path, respectively. The acoustic impedance is described as,

$$
Z=\rho \times c
$$

where $\rho$ is the density of air, and $\mathrm{c}$ is the velocity of sound in air. As the beam propagates through the air, it generates an optically-induced depletion layer, due to excited gas molecules in the beams path undergoing non-radiative relaxation. This optically-induced depletion layer [see Figure 1] has a significantly different acoustic impedance than outside, resulting in efficient reflection of acoustic waves impinging on the THORS barrier. 


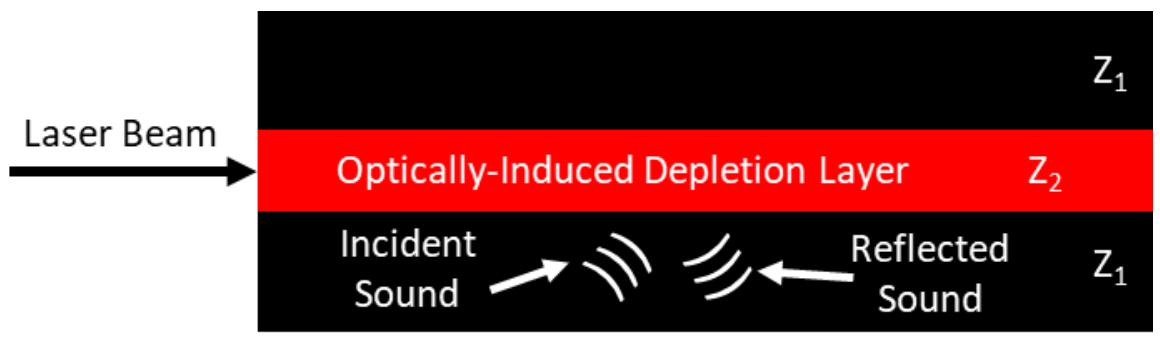

Figure 1. A THORS barrier is generated by optically depleting the air in the path of the beam and producing an abrupt change in compressibility between the inside and the outside of the beams path.

\section{EXPERIMENTAL}

Optically-induced acoustic barriers in air are generated using a waveguide-based $\mathrm{CO}_{2}$ laser (Laser Photonics; Model CL55WTVO) operating at $9.6 \mu \mathrm{m}$ and $3.0 \mathrm{~W}$. The laser is modulated at various frequencies from $0-3.1 \mathrm{kHz}$ using an optical chopper (Scitech; Model 300CD) with a 50\% duty cycle. The modulated laser beam is then passed through a $\mathrm{ZnSe}$ window, and into a plexiglass environmental enclosure (48 inches long x 18 inches wide x 18 inches tall), inside of which the air is saturated with ethanol vapor. A constant source of alcohol vapor was provided by placing a recrystallization dish containing a reservoir of 200-proof ethanol in the bottom of the chamber and allowing it to evaporate for continuous saturation over extended periods of time (i.e., 12 - 15 hours).

Acoustic waveguiding channels using THORS are generated using the previously described $\mathrm{CO}_{2}$ laser-based setup in the ethanol environmental chamber by passing the modulated beam through a ZnSe beam expander (II-VI Inc.; Model BEC210.6C1.05-D2) [see Figure 2] prior to passing into the chamber through the ZnSe window. The beam is expanded

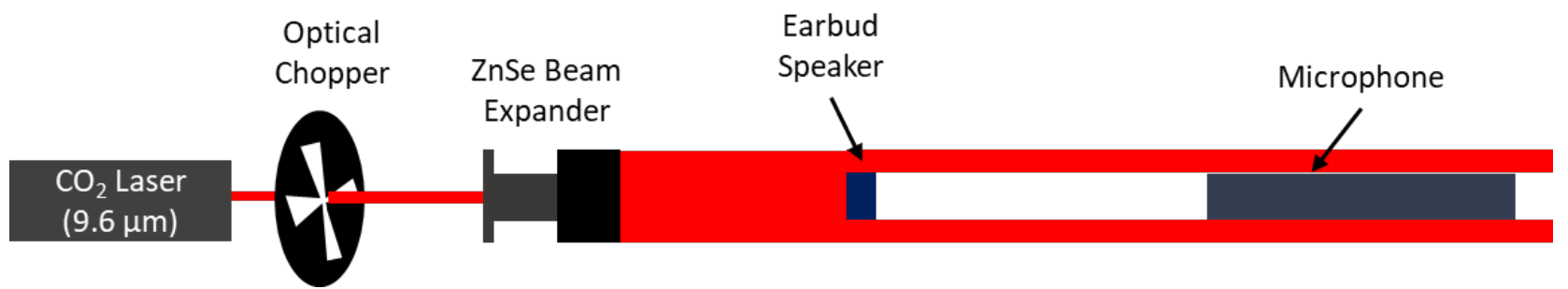

Figure 2. The experimental setup for performing THORS channeling.

to approximately 1-inch in diameter. Inside the chamber, the expanded beam was shaped into a donut-shaped channel by masking the center of the beam with an external sound source (i.e. earbud; JLabs; Model J6M), facing downfield of the beam. A variable frequency function generator (GW Instek; Model GFG-3015) was connected to the acoustic source to produce controlled monotonic frequency soundwaves ranging from $2 \mathrm{kHz}$ to $20 \mathrm{kHz}$ (the audible frequency range). Measurement of the acoustic signal amplitude was performed using a 0.5 -inch nominal diameter, free field $0^{\circ}$ microphone (ACO Pacific; Model No. 7046) coupled to a preamplifier (ACO Pacific; Model No. 4012). The microphone was placed inside the channel and faced directly at the earbud speaker. The TTL output from the optical chopper and the output of the microphone were monitored using a 500-MHz digital oscilloscope (LeCroy; Model Waverunner LT342).

\section{RESULTS AND DISCUSSION}

\subsection{THORS acoustic waveguiding channels}

In previous work, the acoustic decay profile for a fixed frequency sound wave emitted down the path of the channel by the earbud speaker was determined by measuring the acoustic amplitude of the wave as a function of distance from the source. ${ }^{7}$ Without the channel, the acoustic amplitude decayed at a rate of $1 / \mathrm{r}$ with distance, as expected based on classical acoustics of soundwaves propagating in air. When the THORS channel was employed however, the acoustic amplitude decayed at 
a significantly slower rate of $1 / \mathrm{r}^{0.6}$ with distance. The acoustic decay rate can be used to determine the acoustic amplitude of a wave over distance with

$$
L_{2}=L_{1}-\left|20 \times \log \left(\frac{r_{1}^{0.6}}{r_{2}^{0.6}}\right)\right|
$$

where $\mathrm{L}_{1}$ and $\mathrm{L}_{2}$ are the sound levels $(\mathrm{dB})$ of the starting distance and the final distance of a propagating sound wave from the source, respectively. $\mathrm{r}_{1}$ and $r_{2}$ are the starting and final distances of a propagating sound wave from the source, respectively. As an example, we can take a 70-dB sound wave (the approx. acoustic amplitude of city traffic) heard at 1meter away and calculate the acoustic amplitude at $1 \mathrm{~km}$ with both the $1 / \mathrm{r}$ and $1 / \mathrm{r}^{0.6}$ decay profiles. Using the traditional $1 / \mathrm{r}$ decay profile, that $70-\mathrm{dB}$ sound wave would dampen to a sound level of $10 \mathrm{~dB}$ (the sound of a pin dropping). When employing a THORS channel and achieving a $1 / \mathrm{r}^{0.6}$ decay profile however, the $70-\mathrm{dB}$ sound wave would dampen to a sound level of $34-\mathrm{dB}$ (the sound of a light rainfall).

When employing THORS acoustic waveguiding channels, the ring-shaped optically-induced depletion layer generates separate acoustic impedances, where the $Z_{1}$ and $Z_{2}$ still represent the acoustic impedance outside and inside the beams path, respectively. A third acoustic impedance, $Z_{3}$, however is generated inside the THORS channel [see Figure 3],

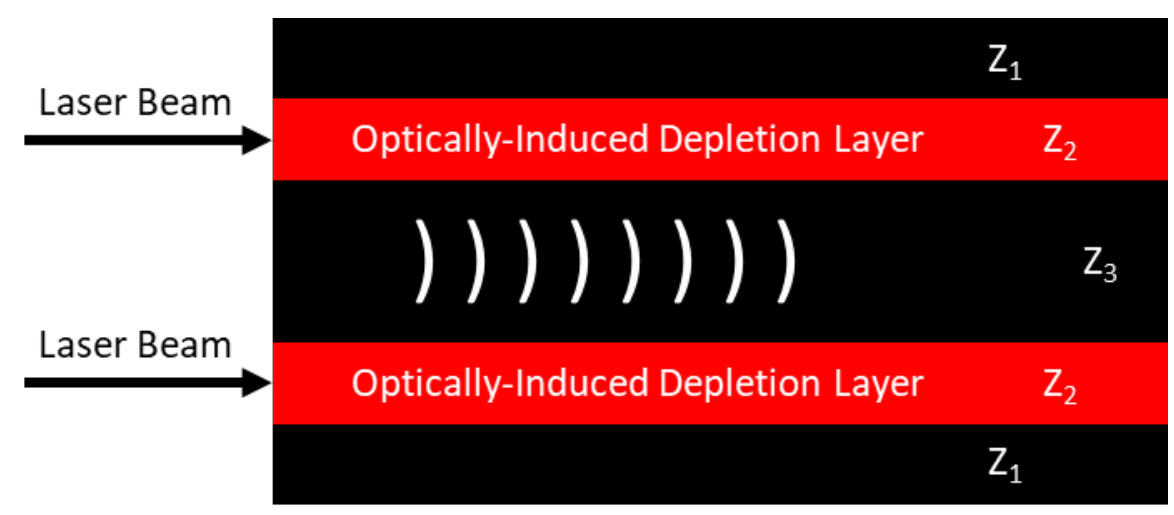

Figure 3. 2-dimensional depiction of a THORS acoustic waveguiding channel. The optically-induced depletion layer wraps around the internally propagating sound waves, providing a reflective barrier that prevents the soundwaves from escaping, thereby extending the propagation distance of the sound.

where the air from the optically-induced depletion layer compresses the air inside the channel. Because of this compression, the difference between $Z_{2}$ and $Z_{3}$ are even greater than the difference between $Z_{1}$ and $Z_{2}$, further increasing the acoustic reflection of incident soundwaves.

\subsection{Extending propagation distance and suppression of external waves}

In order to increase the sensitivity of photoacoustic signal detection at extended distances, external background noise should also be suppressed, thereby further increasing the signal-to-background and signal-to-noise ratios. To demonstrate the potential for THORS to both extend the propagation distance of acoustic waves, while simultaneously suppressing background noise, a second earbud speaker was incorporated in the THORS channeling setup previously discussed. The second earbud was placed outside the channel and angled toward the microphone at an equivalent distance to the earbud inside the channel. Both earbuds were controlled with an iPhone 6s Plus function generator application (f Generator PRO; V4.5.0[1113]). The external earbud emitted two frequencies $(7.1 \mathrm{kHz}$ and $7.3 \mathrm{kHz})$ while the inside-channel earbud emitted a single frequency at $7.0 \mathrm{kHz}$.

All three frequencies signal amplitudes were monitored on the digital oscilloscope and transformed into the Fourier domain, to distinguish their respective magnitudes. Measurements were taken both when the $\mathrm{CO}_{2}$ laser was on and when it was off to determine the enhancement of the internal $7.0 \mathrm{kHz}$ signal and suppression of externally propagating $7.1 \mathrm{kHz}$ and $7.3 \mathrm{kHz}$ frequency sound waves [see Figure 4]. The reference amplitudes (top spectrum; Fig 4) of the three acoustic 
frequencies are taken when there is no THORS channel present. Once the THORS channel is turned on, and we monitor the acoustic amplitudes of the three frequencies (bottom spectrum, Fig 4), we can see the change in amplitude for both the internally guided waves and external acoustic waves. The $7.0 \mathrm{kHz}$ wave emitted inside the channel increased in amplitude by $33 \% \pm 8 \%$. Alternatively, the outside frequencies of $7.1 \mathrm{kHz}$ and $7.3 \mathrm{kHz}$ were both suppressed, $39 \% \pm 10 \%$ and $59 \%$ $\pm 3 \%$, respectively, demonstrating the ability to dramatically improve signal transmission in the presence of background noise from spatially isolated sources.

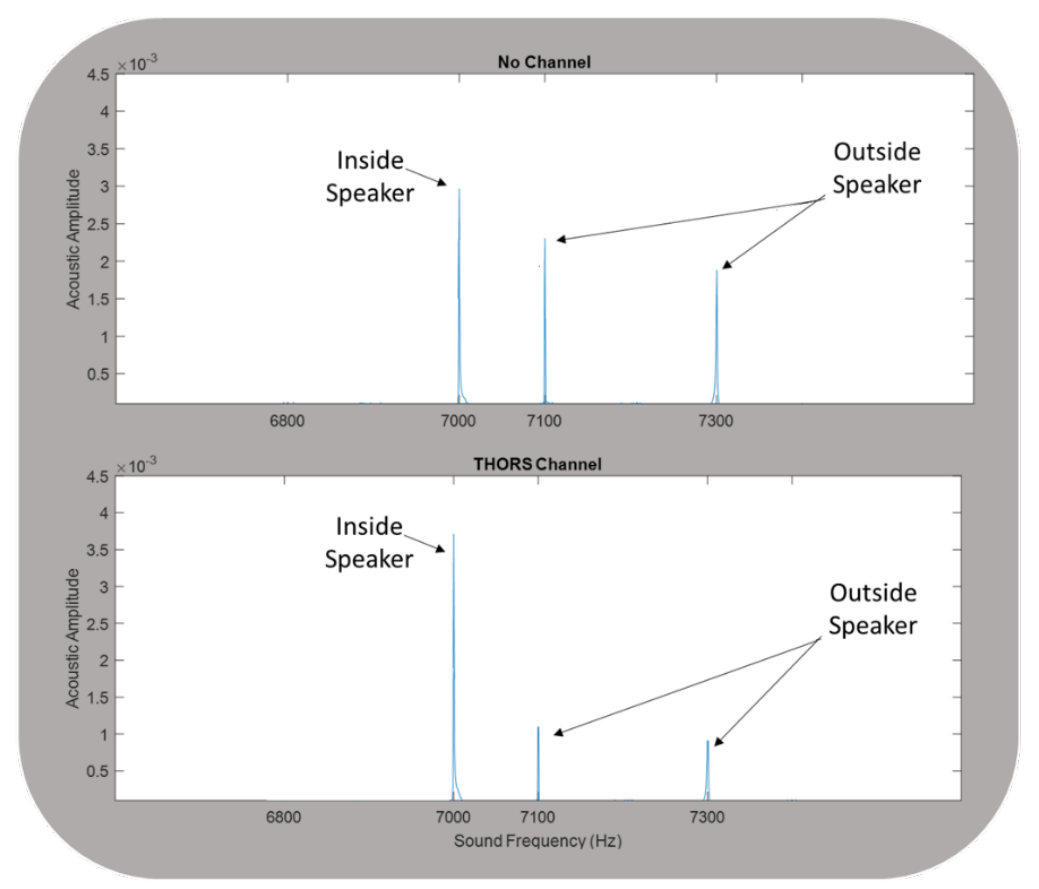

Figure 4. The acoustic amplitudes of the THORS channel internal source $(7.0 \mathrm{kHz})$ and external source $(7.1 \mathrm{and} 7.3 \mathrm{kHz})$ are shown with no channel present (top) and when the THORS channel is employed (bottom).

\subsection{Optical modulation frequency effect on THORS channeling}

From previous studies involving THORS reflection off of isolated barriers, it was seen that a critical optical modulation frequency is necessary to generate an efficient barrier. To determine whether or not a similar relationship between optical modulation frequency and acoustic waveguiding efficiency occur, we performed a similar study. In this study, a microphone was placed at a distance of 14 inches from the earbud speaker, emitting a fixed $7.0 \mathrm{kHz}$ frequency soundwave generated by an Instek function generator. Both the TTL signal from the optical chopper and acoustic wave were monitored on the digital oscilloscope. The optical modulation frequency was then varied between a continuous wave beam and 2400 $\mathrm{Hz}$ in $200 \mathrm{~Hz}$ increments. The amplitude of the sinusoidal soundwave was measured at each frequency under the TTL laser on (channel present) and laser off (no channel) portions of the square wave. To determine the efficiency of THORS channeling, the difference in amplitude of the two signals were recorded [see Figure 5]. The previous work that evaluated THORS reflection and suppression of soundwaves incident to the barrier demonstrated that a minimum $500 \mathrm{~Hz}$ optical modulation frequency was necessary, in order to achieve efficient reflection of sound. In this work with THORS channeling of sound, similar results were observed. When the optical modulation frequency reaches $500 \mathrm{~Hz}$, a rapid increase in the signal amplitude of soundwaves propagating inside the THORS channel is observed, that quickly levels off at higher modulation frequencies. At optical modulation frequencies of $500 \mathrm{~Hz}$ or lower, no significant waveguiding occurs. 


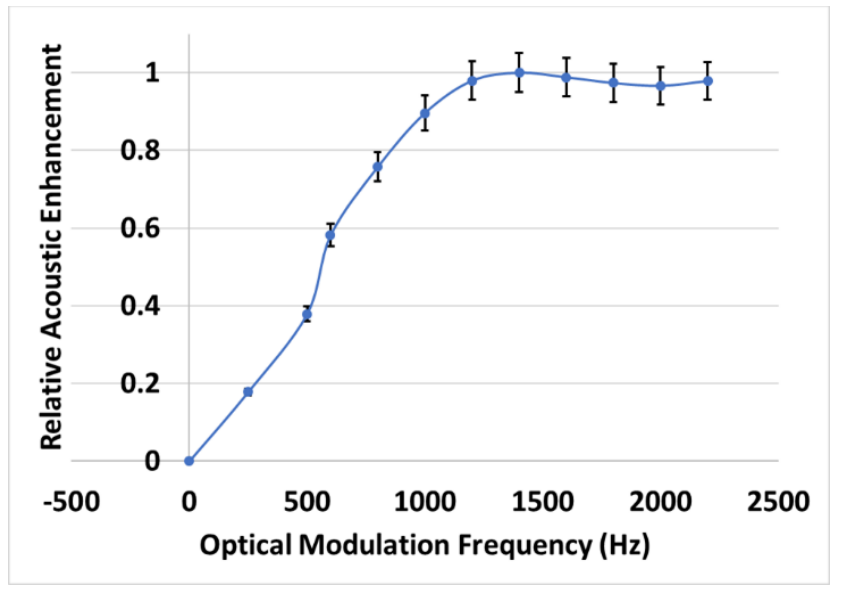

Figure 5. The relative acoustic enhancement of a $7 \mathrm{kHz}$ sound wave propagating down a THORS channel as a function of optical modulation frequency.

\section{CONCLUSION}

In summary, we have demonstrated in this work that THORS can be used as an effective means of acoustically waveguiding sound waves over extended distances. Similarly, the ability to spatially discriminate between signals being waveguided offers the potential for enhanced spatial resolution in photoacoustic imaging studies as well. In order to achieve the most efficient THORS channeling, the optical modulation frequency of $\mathrm{CO}_{2}$ laser must be $1000 \mathrm{~Hz}$ or greater. Furthermore, due to the abrupt difference in acoustic impedance on the outside of a THORS channel and the ambient air, external acoustic waves incident to the THORS channel are suppressed to increase the signal-to-noise and signal-to-background ratios of the internal soundwave. The employment of higher power laser sources and different optical geometries for THORS channeling could allow for even greater efficiencies to be achieved, extending acoustic wave propagation distances even further. This ability to generate optically-induced acoustic waveguides in samples provides the potential to enhance photoacoustic signal propagation distances for use in various applications.

\section{REFERENCES}

[1] Tyndall J. Sound: A Course of Eight Lectures Delivered at the Royal Institution of Great Britain. Cambridge: Cambridge University Press, (2014).

[2] Strutt JW. "The scientific work of Tyndall," Proc. Royal Inst. of Great Britain Proc. Royal Inst. of Great Britain 14 216-224 (1894).

[3] Bell AG. "On the production and reproduction of sound by light," Am. J. Sci. Am. J. Sci. 20 (118), 305-324 (1880).

[4] Bell AG. "The production of sound by radiant energy," Science Science 2 (49), 242-253 (1881).

[5] Haisch C, Niessner R. Light and sound - Photoacoustic spectroscopy. (2002).

[6] Rosencwaig A. Photoacoustics and photoacoustic spectroscopy / Allan Rosencwaig. (1982).

[7] Cullum BM, Holthoff EL, Pellegrino PM. "Optical reflection and waveguiding of sound by photo-thermally induced barriers," Opt. Express Opt. Express 25 (19), 22738-22749 (2017). 